Editorial

\title{
Coatings Best Paper Award 2016
}

\author{
Alessandro Lavacchi \\ Editor-in-Chief of Coatings, Istituto di Chimica dei Composti OrganoMetallici (ICCOM-CNR), \\ Via Madonna del Piano 10, 50019 Sesto Fiorentino, Firenze, Italy; alessandro.lavacchi@iccom.cnr.it \\ Received: 1 September 2016; Accepted: 1 September 2016; Published: 2 September 2016
}

Coatings has established the Best Paper Award to recognize its best published articles each year. The prize started in 2015 [1], and as of 2016, it has awarded in two categories: Best Original Research Article and Best Review Paper.

Nominations were selected by the Editorial Office of Coatings among the Papers published in 2015 , with a selection process based on the number of citations and downloads from the website.

The selection committee-which consisted of three members of the Editorial Board and the Editor in Chief-examined each paper and expressed their preferences, releasing the ranking of the "Coatings Best Paper Award" 2016.

Original Research Article Award:

1st Prize

Performance Testing of Suspension Plasma Sprayed Thermal Barrier Coatings Produced with Varied Suspension Parameters

Nicholas Curry, Kent VanEvery, Todd Snyder, Johann Susnjar and Stefan Bjorklund

Coatings 2015, 5(3), 338-356; doi:10.3390/coatings5030338

Available online: http://www.mdpi.com/2079-6412/5/3/338

2nd Prize

Preservation of Historical Stone Surfaces by $\mathrm{TiO}_{2} \mathrm{Nanocoatings}$

Giovanni Battista Goffredo and Placido Munafò

Coatings 2015, 5(2), 222-231; doi:10.3390/coatings5020222

Available online: http:/ /www.mdpi.com/2079-6412/5/2/222

3rd Prize

High Energy Radial Deposition of Diamond-Like Carbon Coatings

Konrad Suschke, René Hübner, Peter Paul Murmu, Prasanth Gupta, John Futter and Andreas Markwitz Coatings 2015, 5(3), 326-337; doi:10.3390/coatings5030326

Available online: http://www.mdpi.com/2079-6412/5/3/326

Review Paper Award:

Bio-Based Coatings for Paper Applications

Vibhore Kumar Rastogi and Pieter Samyn

Coatings 2015, 5(4), 887-930; doi:10.3390/coatings5040887

Available online: http:/ /www.mdpi.com/2079-6412/5/4/887 
These four outstanding papers are valuable contributions to Coatings. On behalf of the Prize Awarding Committee and the Editorial Board, we would like to congratulate these four teams for their excellent work. In recognition for their accomplishment, Dr. Nicholas Curry, Dr. Giovanni Battista Goffredo, Dr. Andreas Markwitz, and Dr. Pieter Samyn will received the privilege to publish an additional paper free of charge in open access format in Coatings after the usual peer-review procedure.

\section{Prize Awarding Committee}

\section{Editor-in-Chief}

Dr. Alessandro Lavacchi

Istituto di Chimica dei Composti OrganoMetallici (ICCOM-CNR), Via Madonna del Piano 10, 50019 Sesto Fiorentino, Firenze, Italy

Editorial Board Member

Prof. Dr. Petri Vuoristo

Surface Engineering Laboratory, Department of Materials Science, Tampere University of Technology, FIN-33101 Tampere, Finland

Editorial Board Member

Prof. Dr. Philippe Dubois

Center of Innovation and Research in Materials \& Polymers, University of Mons, Place du Parc, 237000 Mons, Belgium

Editorial Board Member

Prof. Dr. Robert B. Heimann

Am Stadtpark 2A, D-02826 Goerlitz, Germany

\section{Reference}

1. Lavacchi, A. Coatings Best Paper Award 2015. Coatings 2015, 5, 219-221. [CrossRef]

(C) 2016 by the author; licensee MDPI, Basel, Switzerland. This article is an open access article distributed under the terms and conditions of the Creative Commons Attribution (CC-BY) license (http://creativecommons.org/licenses/by/4.0/). 